\title{
RAZUMIKHIN-TYPE THEOREMS OF INFINITE DIMENSIONAL STOCHASTIC FUNCTIONAL DIFFERENTIAL EQUATIONS
}

\author{
Kai Liu ${ }^{1}$ and Yufeng Shi ${ }^{2}$ \\ ${ }^{1}$ Department of Mathematical Sciences, The University of Liverpool, Liverpool, L69 7ZL, $U$. \\ $K .{ }^{*}$ School of Mathematics and System Sciences, Shandong University, Jinan 250100, China, \\ $y f$ shi@sdu.edu.cn ${ }^{\dagger}$
}

\begin{abstract}
The argument of Razumikhin-type has been well developed and showed significant advantage for the stability of stochastic functional differential equations in finite dimensions. However, so far there have been almost no results of Razumikhin-type on the stability of mild solutions of stochastic functional differential equations in infinite dimensions. The main aim of this paper is to establish Razumikhin-type stability theorems for stochastic functional differential equations in infinite dimensions. By virtue of these new criteria, we can establish the exponential stability of stochastic delay differential equations and stochastic delay partial differential equations.
\end{abstract}

Key words: Lyapunov function; Razumikhin-type theorem; Stochastic functional differential equations in infinite dimensions.

\section{Introduction}

Stochastic functional differential equations in infinite dimensional spaces are motivated by the development of analysis and the theory of stochastic processes itself such as stochastic partial differential equations with some hereditary characteristics on the one hand, and by such topics as wave propagation in random media, turbulence, population biology and stochastic control in applications on

\footnotetext{
*This work was supported by EPSRC Grant No. GR/R37227.

Partially supported by Foundation for University Key Teacher by Ministry of Education of China, National Natural Science Foundation of China grant 10201018 and Doctor Promotional Foundation of Shandong grant 02BS127.
}

Please use the following format when citing this chapter:

Liu, K., and Shi, Y., 2006, in IFIP International Federation for Information Processing, Volume 202, Systems, Control, Modeling and Optimization, eds. Ceragioli, F., Dontchev, A., Furuta, H., Marti, K., Pandolfi, L., (Boston: Springer), pp. 237-247. 
the other. The analysis and control of such systems then involve investigating their stability, which is often regarded as the first characteristic of dynamical systems (or models) studied.

The purpose of this paper is to investigate stability of mild solutions for certain infinite dimensional stochastic functional differential equations. Roughly speaking, we shall consider the following stochastic functional differential equations in a certain Hilbert space $H$ with norm $\|\cdot\|_{H}$ :

$\begin{aligned} d u(t) & =A u(t) d t+F\left(t, u(t), u_{t}\right) d t+G\left(t, u(t), u_{t}\right) d W(t), \quad t \geq 0, \\ u_{0} & =\xi \in C_{\mathcal{F}_{0}}^{b}([-r, 0] ; H),\end{aligned}$

where $A$ is the infinitesimal generator of a certain $C_{0}$-semigroup $\{T(t), t \geq 0\}$ of bounded linear operators on $H$ and $F: \mathbf{R}^{+} \times H \times C([-r, 0] ; H) \rightarrow H$ and $G: \mathbf{R}^{+} \times H \times C([-r, 0] ; H) \rightarrow \mathcal{L}(K, H)$ are two measurable nonlinear mappings. Here $K$ is some real separable Hilbert space and $u_{t}=$ $\{u(t+\theta):-r \leq \theta \leq 0\}$ is regarded as a $C([-r, 0] ; H)$-valued stochastic process. The family of all bounded, $\mathcal{F}_{0}$-measurable, $C([-r, 0] ; H)$-valued random variables is denoted $C_{\mathcal{F}_{0}}^{b}([-r, 0] ; H)$. The process $\{W(t)\}_{t \geq 0}$ is some given $K$-valued, $Q$-Wiener process with $\operatorname{tr}(Q)<\infty$ and $\xi(t): \Omega \times[-r, 0] \rightarrow$ $H, r \geq 0$, is a given initial datum such that $\xi(t)$ is $\mathcal{F}_{0}$-measurable and $\sup _{-r \leq t \leq 0} E\|\xi(t)\|_{H}^{2}<\infty$.

Stochastic evolution equations in Hilbert spaces have been studied by many authors over the last several years. For instance, Da Prato and Zabczyk [3] and Pardoux [4] (amongst others) have established results on the existence and uniqueness of solutions for a certain class of infinite dimensional stochastic evolution equations. For variable delay case, the similar problems have been studied by Real [5] for stochastic linear evolution equations and by Caraballo and Liu [6] and Caraballo, Liu and Truman [7] for nonlinear cases. On the other hand, under various circumstances there exists an extensive literature on stability of infinite dimensional stochastic differential equations. In particular, we like to refer to $[2,8]$ on the stability of mild solutions for infinite dimensional stochastic functional differential equations.

In infinite dimensional setting, for the purpose of deriving stability results, a suitable construction of Lyapunov functionals rather than functions is a natural generalization of the Lyapunov direct method in finite dimensional spaces. We present below a Lyapunov functional type of argument of stability to show that the situation in treating (1) by this approach could become very complicated. Suppose $u(t ; \xi)$ is the solution of (1) through $(0, \xi)$ and $u_{t}(\xi)=$ $\{u(t+\theta ; \xi):-r \leq \theta \leq 0\}$. Let us study a typical stability result which is a direct stochastic version of Theorem 2.1 in Chapter 5 in [9]. The reader is referred to [9] for more details.

Proposition 1 Let $p \geq 2$ and the standard hypothesis (H1) imposed in Section 2 hold. Suppose $v(\cdot), l(\cdot): \mathbf{R}^{+} \rightarrow \mathbf{R}^{+}$are two continuous nonde- 
creasing functions, $v(s)$ and $l(s)$ are positive for $s>0, v(s)$ is convex and $l(s)$ is concave with $v(0)=l(0)=0$. If there is a continuous functional $V: \mathbf{R} \times C([-r, 0] ; H) \rightarrow \mathbf{R}$ such that

$$
\begin{aligned}
v\left(\|\varphi(0)\|_{H}^{p}\right) & \leq V(t, \varphi) \leq l\left(\|\varphi\|_{C}^{p}\right), \quad \forall \varphi \in C([-r, 0] ; H) \\
E V\left(t, u_{t}(\xi)\right) & \leq E V\left(s, u_{s}(\xi)\right), \quad \forall t \geq s \geq 0 .
\end{aligned}
$$

Then the (mild) solution of (1) is p-th moment stable.

In spite of the formal simplicity of the above result, it is hard to apply this proposition directly to practical problems even though $H$ is finite dimensional, e.g., $H=\mathbf{R}^{n}$. The reason is twofold. On the one hand, instead of the usual Lyapunov functions in finite dimensional spaces, a Lyapunov functional as above must be constructed properly, a case which is usually not easy to handle. On the other hand, the conditions as above are difficult to justify because of the inclusion of the solution itself which is not known explicitly in most situations. This proposition certainly loses the advantage of the Lyapunov direct method in the sense that it is unnecessary to solve the equations explicitly in order to determine the stability of solutions.

One of the most effective ways to deal with these problems is a method originated by Razumikhin $[10,11]$. The argument of Razumikhin-type has been well developed and showed significant advantage for the stability of (stochastic) functional differential equations in finite dimensions (see $[9,12,13]$ ). The Lyapunov functions of Razumikhin-type have been shown to be rather powerful to treat functional differential equations, and as a consequence, that they really bring forth the advantage of Lyapunov direct method. In [13] Mao has shown a smart argument of Razumikhin-type to exponential stability of finite dimensional stochastic functional differential equations. However by virtue of Mao's argument, it is not trivial to treat the case in infinite dimensions, because there is not Itô's formula applicable to mild solutions of stochastic functional differential equations in infinite dimensions. So far there have been almost no results of Razumikhin-type on the stability of mild solutions of infinite dimensional stochastic functional differential equations. To the best of our knowledge this is the first time the possibility of using Lyapunov functions of Razumikhin-type to determine sufficient conditions of stability for stochastic functional differential equations in infinite dimensions has been explored. By virtue of the new criteria derived later on, we can show the exponential stability of stochastic delay differential equations and stochastic delay partial differential equations. In particular, by using the results derived in this paper we may essentially improve some stability results in $[1,2]$. 


\section{Preliminary results}

Let $\left(\Omega, \mathcal{F},\left\{\mathcal{F}_{t}\right\}_{t>0}, P\right)$ be a complete probability space with a filtration $\left\{\mathcal{F}_{t}\right\}_{t>0}$ satisfying the usual conditions (i.e., it is right continuous and $\mathcal{F}_{0}$ contains all $P$-null sets). Let $K$ be a real separable Hilbert space. With the symbol $\{W(t), t \geq 0\}$ we denote a $K$-valued $\left\{\mathcal{F}_{t}\right\}_{t \geq 0}$-Wiener process defined on the probability space $\left(\Omega, \mathcal{F},\left\{\mathcal{F}_{t}\right\}_{t \geq 0}, P\right)$ with covariance operator $Q$, i.e.,

$$
E(W(t), x)_{K}(W(s), y)_{K}=(t \wedge s)(Q x, y)_{K} \forall x, y \in K,
$$

where $Q$ is a nonnegative finite trace class operator from $K$ into itself. In particular, we call $\{W(t)\}_{t>0}$ a $K$-valued $Q$-Wiener process with respect to $\left\{\mathcal{F}_{t}\right\}_{t \geq 0}$.

Let $H$ be a real Hilbert space and we denote by $\langle\cdot, \cdot\rangle$ its inner product and $\|\cdot\|_{H}$ its norm, respectively. Assume $r$ is a given positive constant. In the present paper, we shall consider the following infinite dimensional stochastic functional differential equation on $I=[-r, T]$, (here $T \geq 0$ and $t \in[0, T]$ )

$$
\begin{aligned}
d u(t) & =A u(t) d t+F\left(t, u(t), u_{t}\right) d t+G\left(t, u(t), u_{t}\right) d W(t), \\
u_{0} & =\xi \in C_{\mathcal{F}_{0}}^{b}([-r, 0] ; H) .
\end{aligned}
$$

Throughout this paper, we shall impose the following assumptions:

(H1) $A$ is the infinitesimal generator of a $C_{0}$-semigroup $\{T(t), t \geq 0\}$ of bounded linear operators on $H$ satisfying $\|T(t)\| \leq M \cdot e^{\lambda t}$ for some $M \geq 1, \lambda \in \mathbf{R}^{1}$. The coefficients $F: \mathbf{R}^{+} \times H \times C([-r, 0] ; H) \rightarrow H$ and $G: \mathbf{R}^{+} \times H \times C([-r, 0] ; H) \rightarrow \mathcal{L}(K, H)$ are two measurable nonlinear mappings satisfying the following Lipschitz condition

$$
\begin{array}{r}
\left\|F(t, x, y)-F\left(t, x^{\prime}, y^{\prime}\right)\right\|_{H}+\left\|G(t, x, y)-G\left(t, x^{\prime}, y^{\prime}\right)\right\|_{2} \\
\leq k\left(\left\|x-x^{\prime}\right\|_{H}+\left\|y-y^{\prime}\right\|_{H}\right),
\end{array}
$$

for some constant $k>0$ and arbitrary $x, x^{\prime}, \in H, y, y^{\prime} \in C([-r, 0] ; H)$ and $t \in \mathbf{R}^{+}$. Here $\|\cdot\|_{2}$ denotes the Hilbert-Schmidt norm of a nuclear operator, i.e., $\|G(t, x, y)\|_{2}^{2}=\operatorname{tr}\left(G(t, x, y) Q G(t, x, y)^{*}\right), x \in H, y \in C([-r, 0] ; H)$. Denote by $C_{\mathcal{F}_{0}}^{b}([-r, 0] ; H)$ the family of all bounded, $\mathcal{F}_{0}$-measurable, $C([-r, 0] ; H)$-valued random variables. For $p \geq 2$ and $t \geq 0$, denote by $L_{\mathcal{F}_{t}}^{p}([-r, 0] ; H)$ the family of all $\mathcal{F}_{t}$-measurable $C([-r, 0] ; H)$-valued random variables $\phi=\{\phi(\theta):-r \leq \theta \leq 0\}$ such that

$$
\sup _{-r \leq \theta \leq 0} E\|\phi(\theta)\|_{H}^{p}<\infty .
$$

We introduce two kinds of solutions of (2) as follows similarly to [14]: 
DEFINITION 2 A stochastic process $u(t), t \in I$, is called a strong solution of (2) if (i) $u(t)$ is adapted to $\mathcal{F}_{t}$;

(ii) $u(t)$ is continuous in t almost surely;

(iii) $u(t) \in \mathcal{D}(A)$ on $I \times \Omega$ with $\int_{0}^{T}\|A u(t)\|_{H} d t<\infty$ almost surely and

$$
\begin{aligned}
u(t)= & \xi(0)+\int_{0}^{t}\left(A u(s)+F\left(s, u(s), u_{s}\right)\right) d s+ \\
& \int_{0}^{t} G\left(s, u(s), u_{s}\right) d W(s), \\
u(t)= & \xi(t), \quad t \in[-r, 0],
\end{aligned}
$$

for all $t \in I$ with probability one.

In general, this concept is rather strong and a weaker one described below is more appropriate for practical purposes.

Definition 3 A stochastic process $u(t), t \in I$, is called a mild solution of (2) if (i) $u(t)$ is adapted to $\mathcal{F}_{t}$;

(ii) $u(t)$ is measurable with $\int_{0}^{T}\|u(t)\|_{H}^{2} d t<\infty$ almost surely and

$$
\begin{aligned}
u(t)= & T(t) \xi(0)+\int_{0}^{t} T(t-s) F\left(s, u(s), u_{s}\right) d s \\
& +\int_{0}^{t} T(t-s) G\left(s, u(s), u_{s}\right) d W(s), \\
u(t)= & \xi(t), \quad t \in[-r, 0],
\end{aligned}
$$

for all $t \in I$ with probability one.

Note that if $\{u(t), t \in I\}$ is a strong solution of (2), then it is also a mild solution. The following existence and uniquness theorem can be obtained similarly by an adapted argument from [6] or [15]. The reader is referred to them for further details on this aspect.

THEOREM 4 Let $\{\xi(t), t \in[-r, 0]\}$ be a given $\mathcal{F}_{0}$-measurable initial datum with $\sup _{-r \leq t<0} E\|\xi(t)\|_{H}^{2}<\infty$. Suppose the hypothesis (H1) holds, then (2) has a unique mild solution $u(t ; \xi)$, or simply $u(t)$, in $C\left(0, T ; L^{2}(\Omega, \mathcal{F}, P ; H)\right)$.

For our purposes, we can introduce Itô's formula which will play an important role in our stability analysis as follows.

Let $C^{2}(R \times H)$ denote the space of all real-valued functions $V$ on $\mathbf{R} \times H$ with properties:

(i) $V(t, x) \in C^{2}(\mathbf{R} \times H)$ is twice (Fréchet) differentiable in $x$ and once differentiable in $t$; 
(ii) $V_{x}(t, x)$ and $V_{x x}(t, x)$ are both continuous in $H$ and $\mathcal{L}(H)=\mathcal{L}(H, H)$, respectively.

Theorem 5 (Itô's formula) Suppose $V \in C^{2}(\mathbf{R} \times H)$ and $\{u(t), t \geq 0\}$ is a strong solution of (2), then

$$
\begin{aligned}
V(t, u(t))= & V(0, \xi(0))+\int_{0}^{t} \mathcal{L} V\left(s, u(s), u_{s}\right) d s \\
& +\int_{0}^{t}\left\langle V_{x}(s, u(s)), G\left(s, u(s), u_{s}\right) d W(s)\right\rangle,
\end{aligned}
$$

where $\forall x \in \mathcal{D}(A), \quad y \in C([-r, 0] ; H), \quad t \geq 0$,

$$
\begin{aligned}
\mathcal{L} V(t, x, y)= & V_{t}(t, x)+\left\langle V_{x}(t, x), A x+F(t, x, y)\right\rangle \\
& +\frac{1}{2} \operatorname{tr}\left(V_{x x}(t, x) G(t, x, y) Q G^{*}(t, x, y)\right) .
\end{aligned}
$$

Since Itô's formula is only applicable to strong solutions, we introduce the following approximating systems of (2), for $t \geq 0$,

$$
\begin{aligned}
d u(t)= & A u(t) d t+R(n) F\left(t, u(t), u_{t}\right) d t \\
& +R(n) G\left(t, u(t), u_{t}\right) d W(t), \\
u(t)= & R(n) \xi(t) \in \mathcal{D}(A), \quad t \in[-r, 0]
\end{aligned}
$$

where $n_{0} \leq n \in \rho(A)$, the resolvent set of $A$ and $R(n)=n R(n, A), R(n, A)$ is the resolvent of $A$. A similar operator $\mathcal{L}_{n}$ to (4) in correspondence with this equation is

$$
\begin{aligned}
\mathcal{L}_{n} V(t, x, y)= & V_{t}(t, x)+\left\langle V_{x}(t, x), A x+R(n) F(t, x, y)\right\rangle \\
& +\frac{1}{2} \operatorname{tr}\left(V_{x x}(t, x) R(n) G(t, x, y) Q(R(n) G(t, x, y))^{*}\right), \\
\forall x \in & \mathcal{D}(A), \quad y \in C([-r, 0] ; H), \quad t \geq 0 .
\end{aligned}
$$

THEOREM 6 ([15]) Under the hypotheses of Theorem 4, (5) has a unique strong solution $u^{n}(t)$ in $C\left(0, T ; L^{2}(\Omega, \mathcal{F}, P ; H)\right)$ for all $T \geq 0$. Moreover, $u^{n}(t)$ converges to the mild solution $u(t)$ of $(2)$ in $C\left(0, T ; L^{2}(\Omega, \mathcal{F}, P ; H)\right)$ as $n \rightarrow \infty$, i.e.,

$$
\lim _{n \rightarrow \infty} E\left(\sup _{t \in[0, T]}\left\|u^{n}(t)-u(t)\right\|_{H}^{2}\right)=0 .
$$

\section{The main results}

In this section, we shall carry out an argument of Razumikhin type to study the stability of the mild solutions of (2) in the sense of $p$-th moment and pathwise 
with probability one. Based on the ideas of constructing Lyapunov functions rather than functionals, we study the stability of the equation (2) in the spirit of Razumikhin in finite dimensions.

Let $C^{1,2}\left([-r, \infty) \times H ; \mathbf{R}^{+}\right)$denote the family of all nonnegative functions $V(t, x)$ on $[-r, \infty) \times H$ which are continuously twice Fréchet differentiable in $x$ and once differentiable in $t$. Then for $V \in C^{1,2}\left([-r, \infty) \times H ; \mathbf{R}^{+}\right)$, we can introduce, similarly to (4), an operator $\mathcal{L}$ on $C^{1,2}\left([-r, \infty) \times H ; \mathbf{R}^{+}\right)$by

$$
\begin{aligned}
& \mathcal{L} V(t, \varphi(0), \varphi) \doteq V_{t}(t, \varphi(0))+\left\langle V_{x}(t, \varphi(0)), A \varphi(0)\right. \\
& +F(t, \varphi(0), \varphi)\rangle_{H}+, \\
& +\frac{1}{2} \operatorname{tr}\left[V_{x x}(t, \varphi(0)) G(t, \varphi(0), \varphi) Q G^{*}(t, \varphi(0), \varphi)\right], \varphi(0) \in \mathcal{D}(A) .
\end{aligned}
$$

Here $t>0$. We furthermore assume that $F(t, 0,0) \equiv 0$ and $G(t, 0,0) \equiv 0$, we can assert the following the exponential stability for (2).

THEOREM 7 Let the standard hypothesis (HI) hold and $p \geq 2, \lambda, c, c_{1}$ all be positive numbers and $q>1$. If there exists a continuous function $V \in$ $C^{1,2}\left([-r, \infty) \times H ; \mathbf{R}^{+}\right)$satisfying

$$
\begin{aligned}
c\|x\|_{H}^{p} & \geq V(t, x)+\|x\|_{H}\left\|V_{x}(t, x)\right\|_{H}+\|x\|_{H}^{2}\left\|V_{x x}(t, x)\right\|_{H} \\
c_{1}\|x\|_{H}^{p} & \leq V(t, x), \quad \forall(t, x) \in[-r, \infty) \times H,
\end{aligned}
$$

and for arbitrary $t \geq 0$

$$
E \mathcal{L} V(t, \phi(t), \phi) \leq-\lambda E V(t, \phi(0)),
$$

whenever $\phi=\{\phi(\theta):-r \leq \theta \leq 0\} \in L_{\mathcal{F}_{t}}^{p}([-r, 0] ; \mathcal{D}(A))$ satisfies

$$
E V(t+\theta, \phi(\theta))<q E V(t, \phi(0)) \text {, for all }-r \leq \theta \leq 0 .
$$

Here for $\phi \in L_{\mathcal{F}_{t}}^{p}([-r, 0] ; \mathcal{D}(A)), \quad \phi(0) \in \mathcal{D}(A), \quad t \geq 0$

$$
\begin{aligned}
\mathcal{L} V(t, \phi(t), \phi) \doteq & V_{t}(t, \phi(0))+\left\langle V_{x}(t, \phi(0)), A \phi(0)+F(t, \phi(0), \phi)\right\rangle \\
& +\frac{1}{2} \operatorname{tr}\left[V_{x x}(t, \phi(0)) G(t, \phi(0), \phi) Q G^{*}(t, \phi(0), \phi)\right] .
\end{aligned}
$$

Then the mild solution $u(t)$ of (2) is p-th moment exponentially stable, i.e., for all $\xi \in C_{\mathcal{F}_{0}}^{b}([-r, 0] ; H)$

$$
E\|u(t ; \xi)\|_{H}^{p} \leq \frac{c}{c_{1}} E\|\xi\|_{C}^{p} e^{-\gamma t}, \quad \forall t \geq 0,
$$

where $\gamma=\min \left\{\lambda, \frac{\log (q)}{r}\right\}$. 
Proof. Fix the initial data $\xi \in C_{\mathcal{F}_{0}}^{b}([-r, 0] ; H)$ arbitrarily and write $u(t ; \xi)=$ $u(t)$ simply. Let $\varepsilon \in(0, \gamma)$ be arbitrary and set $\bar{\gamma}=\gamma-\varepsilon$. Define

$$
U(t)=\max _{-r \leq \theta \leq 0}\left[e^{\bar{\gamma}(t+\theta)} E V(t+\theta, u(t+\theta))\right], \quad \text { for } t \geq 0 .
$$

Obviously $U(t)$ is well defined and continuous. We can claim that

$$
D^{+} U(t) \doteq \limsup _{h \rightarrow 0+} \frac{U(t+h)-U(t)}{h} \leq 0, \quad \forall t \geq 0 .
$$

To show this, for each fixed $t_{0} \geq 0$, define

$$
\bar{\theta}=\max \left\{\theta \in[-r, 0]: U\left(t_{0}\right)=e^{\bar{\gamma}\left(t_{0}+\theta\right)} E V\left(t_{0}+\theta, u\left(t_{0}+\theta\right)\right)\right\} .
$$

Obviously, $\bar{\theta}$ is well defined, $\bar{\theta} \in[-r, 0]$ and

$$
U\left(t_{0}\right)=e^{\bar{\gamma}\left(t_{0}+\bar{\theta}\right)} E V\left(t_{0}+\bar{\theta}, u\left(t_{0}+\bar{\theta}\right)\right), \text { a.s.. }
$$

If $\bar{\theta}<0$, for all $\bar{\theta}<\theta \leq 0$ one has

$$
e^{\overline{\gamma\left(t_{0}+\theta\right)}} E V\left(t_{0}+\theta, u\left(t_{0}+\theta\right)\right)<e^{\bar{\gamma}\left(t_{0}+\bar{\theta}\right)} E V\left(t_{0}+\bar{\theta}, u\left(t_{0}+\bar{\theta}\right)\right) .
$$

It is therefore easy to observe that for any $h>0$ small enough

$$
e^{\bar{\gamma}\left(t_{0}+h\right)} E V\left(t_{0}+h, u\left(t_{0}+h\right)\right) \leq e^{\bar{\gamma}\left(t_{0}+\bar{\theta}\right)} E V\left(t_{0}+\bar{\theta}, u\left(t_{0}+\bar{\theta}\right)\right)
$$

Hence

$$
U\left(t_{0}+h\right) \leq U\left(t_{0}\right) \text { and } D^{+} U\left(t_{0}\right) \leq 0
$$

If $\bar{\theta}=0$, then

$$
e^{\bar{\gamma}\left(t_{0}+\theta\right)} E V\left(t_{0}+\theta, u\left(t_{0}+\theta\right)\right) \leq e^{\bar{\gamma} t_{0}} E V\left(t_{0}, u\left(t_{0}\right)\right), \quad \forall \theta \in[-r, 0] .
$$

So

$$
\begin{aligned}
E V\left(t_{0}+\theta, u\left(t_{0}+\theta\right)\right) & \leq e^{-\bar{\gamma} \theta} E V\left(t_{0}, u\left(t_{0}\right)\right) \\
& \leq e^{\bar{\gamma} r} E V\left(t_{0}, u\left(t_{0}\right)\right), \quad \forall \theta \in[-r, 0] .
\end{aligned}
$$

Note that either $E V\left(t_{0}, u\left(t_{0}\right)\right)=0$ or $E V\left(t_{0}, u\left(t_{0}\right)\right)>0$. In the case of $E V\left(t_{0}, u\left(t_{0}\right)\right)=0$, (6) and (9) imply that $u\left(t_{0}+\theta\right)=0$ a.s. for all $\theta \in[-r, 0]$. Recalling the fact that $F\left(t_{0}, 0,0\right) \equiv 0$ and $G\left(t_{0}, 0,0\right) \equiv 0$, it follows that $u\left(t_{0}+h\right)=0$ a.s. for all $h>0$, hence $U\left(t_{0}+h\right)=0$ and $D^{+} U\left(t_{0}\right)=0$. On the other hand, in the case of $E V\left(t_{0}, u\left(t_{0}\right)\right)>0$, (9) implies

$$
\begin{aligned}
E V\left(t_{0}+\theta, u\left(t_{0}+\theta\right)\right) & \leq e^{\bar{\gamma} r} E V\left(t_{0}, u\left(t_{0}\right)\right) \\
& <q E V\left(t_{0}, u\left(t_{0}\right)\right), \quad \forall \theta \in[-r, 0],
\end{aligned}
$$


since $e^{\bar{\gamma} r}<q$. Let $\beta=q-e^{\bar{\gamma} r}>0$. It follows from the continuity of $E V\left(t_{0}, u\left(t_{0}\right)\right)$ and (6) that for some $h>0$ sufficiently small

$$
E V\left(t_{0}+\theta, u\left(t_{0}+\theta\right)\right) \leq\left(e^{\bar{\gamma} r}+\frac{\beta}{2}\right) E V\left(t_{0}, u\left(t_{0}\right)\right), \quad \forall \theta \in[0, h] .
$$

Now we need to introduce the strong solutions $u^{n}(t)$ of (5). By virtue of $(\mathrm{H} 1)$, (6) and Theorem 6, there exists a sub-sequence of $\{n\}$ in $\rho(A)$ (still denote by $\{n\})$ such that $u^{n}(t) \rightarrow u(t)$ almost surely as $n \rightarrow \infty$ in $C(0, T ; H)$ uniformly with respect to $t \in[0, T]$. Consequently, for some positive constant $\delta \in\left(0, \frac{\beta}{4+2 \beta} E V\left(t_{0}, u\left(t_{0}\right)\right)\right)$, there are a sufficiently small constant $h>0$ and a large number $N>0$ such that for $n \geq N$, one has that for any $s \in\left[t_{0}, t_{0}+h\right]$

$$
\begin{aligned}
E V(s, u(s)) & >E V\left(t_{0}, u\left(t_{0}\right)\right)-\delta>0, \\
E V(s+\theta, u(s+\theta)) & <E V\left(t_{0}+\theta, u\left(t_{0}+\theta\right)\right)+\delta, \quad \forall \theta \in[-r, 0], \\
e^{\bar{\gamma} r} E V\left(t_{0}, u\left(t_{0}\right)\right) & <e^{\bar{\gamma} r} E V(s, u(s))+\delta, \\
E V\left(s, u^{n}(s)\right) & >E V(s, u(s))-\delta>0, \\
e^{\bar{\gamma} r} E V(s, u(s)) & <e^{\bar{\gamma} r} E V\left(s, u^{n}(s)\right)+\delta, \\
E V\left(s+\theta, u^{n}(s+\theta)\right) & <E V(s+\theta, u(s+\theta))+\delta, \quad \forall \theta \in[-r, 0],
\end{aligned}
$$

which immediately imply

$$
E V\left(s+\theta, u^{n}(s+\theta)\right)<q E V\left(s, u^{n}(s)\right), \quad \forall \theta \in[-r, 0] .
$$

By the condition (7), (10) implies that

$$
\operatorname{EL} V\left(s, u^{n}(s), u_{s}^{n}\right) \leq-\lambda E V\left(s, u^{n}(s)\right), \quad \forall s \in\left[t_{0}, t_{0}+h\right] .
$$

Applying Itô's formula to the function $e^{\bar{\gamma} t} V(t, u)$ along the strong solutions $u^{n}(t)$ of (5), one can derive that for any $\bar{h} \in[0, h]$,

$$
\begin{aligned}
& e^{\bar{\gamma}\left(t_{0}+\bar{h}\right)} E V\left(t_{0}+\bar{h}, u^{n}\left(t_{0}+\bar{h}\right)\right)-e^{\bar{\gamma} t_{0}} E V\left(t_{0}, u^{n}\left(t_{0}\right)\right) \\
& =\int_{t_{0}}^{t_{0}+\bar{h}} e^{\bar{\gamma} s}\left[\bar{\gamma} E V\left(s, u^{n}(s)\right)+E \mathcal{L}_{n} V\left(s, u^{n}(s), u_{s}^{n}\right)\right] d s \\
& =\int_{t_{0}}^{t_{0}+\bar{h}} e^{\bar{\gamma} s}\left[\bar{\gamma} E V\left(s, u^{n}(s)\right)+E \mathcal{L} V\left(s, u^{n}(s), u_{s}^{n}\right)\right] d s \\
& +\int_{t_{0}}^{t_{0}+\bar{h}} e^{\bar{\gamma} s} E\left\langle V_{x}\left(s, u^{n}(s)\right),(R(n)-I) F\left(s, u^{n}(s), u_{s}^{n}\right)\right\rangle d s \\
& +\frac{1}{2} \int_{t_{0}}^{t_{0}+\bar{h}} e^{\bar{\gamma} s} E \operatorname{tr}\left[V_{x x}\left(s, u^{n}(s)\right) R(n) G\left(s, u^{n}(s), u_{s}^{n}\right)\right. \\
& \left.Q\left(R(n) G\left(s, u^{n}(s), u_{s}^{n}\right)\right)^{*}\right] d s \\
& -\frac{1}{2} \int_{t_{0}}^{t_{0}+\bar{h}} e^{\bar{\gamma} s} \operatorname{Etr}\left[V_{x x}\left(s, u^{n}(s)\right) G\left(s, u^{n}(s), u_{s}^{n}\right) Q G^{*}\left(s, u^{n}(s), u_{s}^{n}\right)\right] d s .
\end{aligned}
$$


By virtue of (11), one can deduce

$$
\begin{aligned}
& e^{\bar{\gamma}\left(t_{0}+\bar{h}\right)} E V\left(t_{0}+\bar{h}, u^{n}\left(t_{0}+\bar{h}\right)\right) \\
& \leq e^{\bar{\gamma} t_{0}} E V\left(t_{0}, u^{n}\left(t_{0}\right)\right)+(\bar{\gamma}-\lambda) \int_{t_{0}}^{t_{0}+\bar{h}} e^{\bar{\gamma} s} E V\left(s, u^{n}(s)\right) d s \\
& +\int_{t_{0}}^{t_{0}+\bar{h}} e^{\bar{\gamma} s} E\left\langle V_{x}\left(s, u^{n}(s)\right),(R(n)-I) F\left(s, u^{n}(s), u_{s}^{n}\right)\right\rangle d s \\
& +\frac{1}{2} \int_{t_{0}}^{t_{0}+\bar{h}} e^{\bar{\gamma} s} E \operatorname{tr}\left[V_{x x}\left(s, u^{n}(s)\right) R(n) G\left(s, u^{n}(s), u_{s}^{n}\right)\right. \\
& \left.Q\left(R(n) G\left(s, u^{n}(s), u_{s}^{n}\right)\right)^{*}\right] d s \\
& -\frac{1}{2} \int_{t_{0}}^{t_{0}+\bar{h}} e^{\bar{\gamma} s} E \operatorname{tr}\left[V_{x x}\left(s, u^{n}(s)\right) G\left(s, u^{n}(s), u_{s}^{n}\right) Q G^{*}\left(s, u^{n}(s), u_{s}^{n}\right)\right] d s,
\end{aligned}
$$

which, letting $n \rightarrow \infty$, immediately yields

$$
\begin{aligned}
e^{\bar{\gamma}\left(t_{0}+\bar{h}\right)} E V\left(t_{0}+\bar{h}, u\left(t_{0}+\bar{h}\right)\right) \leq & e^{\bar{\gamma} t_{0}} E V\left(t_{0}, u\left(t_{0}\right)\right) \\
& +\int_{t_{0}}^{t_{0}+\bar{h}}(\bar{\gamma}-\lambda) e^{\bar{\gamma} s} E V(s, u(s)) d s \\
\leq & e^{\bar{\gamma} t_{0}} E V\left(t_{0}, u\left(t_{0}\right)\right) .
\end{aligned}
$$

Then it follows that

$$
e^{\bar{\gamma} s} E V(s, u(s)) \leq e^{\bar{\gamma} t_{0}} E V\left(t_{0}, u\left(t_{0}\right)\right), \quad \forall s \in\left[t_{0}, t_{0}+h\right] .
$$

So it must hold that $E U\left(t_{0}+h\right)=E U\left(t_{0}\right)$ for any $h>0$ sufficiently small, and hence $D^{+} U\left(t_{0}\right)=0$. Since $t_{0}$ is arbitrary, the inequality (8) is shown to hold for any $t \geq 0$. It now follows immediately from (8) that $U(t) \leq U(0)$, for any $t \geq 0$. Also, (6) implies that

$$
e^{\bar{\gamma} t} E V(t, u(t)) \leq U(t) \leq U(0) \leq c E\|\xi\|_{C}^{p}, \quad \forall t \geq 0 .
$$

Note that $\varepsilon$ is arbitrary, it thus follows that

$$
E V(t, u(t)) \leq c E\|\xi\|_{C}^{p} e^{-\gamma t}, \quad \forall t \geq 0,
$$

which, by virtue of (6), immediately yields that

$$
E\|u(t)\|_{H}^{p} \leq \frac{c}{c_{1}} E\|\xi\|_{C}^{p} e^{-\gamma t}, \quad \forall t \geq 0 .
$$

Therefore the desired result is obtained. The proof is complete.

By virtue of the above theorem we can give the almost sure exponential stability for stochastic functional differential equations, similarly to Theorem 2.2 in [13]. We thus omit it at the moment. 


\section{References}

[1] T. Taniguchi. Almost sure exponential stability for stochastic partial functional differential equations. Stoch. Anal. Appl. 16(5):965-975, 1998.

[2] K. Liu, A. Truman. Moment and almost sure Lyapunov exponents of mild solutions of stochastic evolution equations with variable delays via approximation approaches. J. Math. Kyoto Univ. 41:749-768, 2002.

[3] G. Da Prato, J. Zabczyk. Stochastic Equations in Infinite Dimensions. Encyclopedia of Mathematics and its Applications, Cambridge University Press, 1992.

[4] E. Pardoux. Equations aux Dérivées Partielles Stochastiques Nonlinéaires Monotones. Thesis, Université Paris Sud, 1975.

[5] J. Real. Stochastic partial differential equations with delays. Stochastics. 8(2):81-102, 1982-1983.

[6] T. Caraballo, K. Liu. Exponential stability of mild solutions of stochastic partial differential equations with delays. Stoch. Anal. Appl. 17:743-764, 1999.

[7] T. Caraballo, K. Lin, A. Truman. Stochastic functional partial differential equations: existence, uniqueness and asymptotic decay property. Proc. R. Soc. Lond. A 456:1775-1802, 2000 .

[8] T. Taniguchi, K. Liu, A. Truman. Existence, uniqueness and asymptotic behavior of mild solutions to stochastic functional differential equations in Hilbert spaces. J. Diff. Eq. 181:72-91, 2002.

[9] J. K. Hale, S. M. Verduyn Lunel. Introduction to Functional Differential Equations. Springer-Verlag, New York, 1993.

[10] B.S. Razumikhin. On the stability of systems with a delay. Prikl. Mat. Meh. 20:500-512, 1956. (translated into English in J. Appl. Math. Mech.)

[11] B.S. Razumikhin. Applications of Lyapunov's method to problems in the stability of systems with a delay. Automat. i. Telemeh. 21:740-749, 1960. (translated into English in Automat. Remote Control 21:515-520, 1960.)

[12] T. Taniguchi. Moment asymptotic behavior and almost sure Lyapunov exponent of stochastic functional differential equations with finite delays via Lyapunov-Razumikhin method. Stochastics. 58:191-208, 1996.

[13] X. R. Mao. Razumikhin-type theorems on exponential stability of stochastic functional differential equations. Stoch. Proc. Appl. 65:233-250, 1996.

[14] A. Ichikawa. Stability of semilinear stochastic evolution equations. J. Math. Anal. Appl. 90:12-44, 1982.

[15] K. Liu. Lyapunov functionals and asymptotic stability of stochastic delay evolution equations. Stochastics. 63:1-26, 1998. 